\title{
Analysis on the Effect of the Rehabilitation Intervention-Centered Targeted Nursing Model on the Cardiac Function Recovery and Negative Emotions in Patients with Acute Myocardial Infarction
}

\author{
Rong Wang, ${ }^{1,2}$ Gongxiang Duan, ${ }^{2}$ Huilan Xu $\mathbb{D}^{1},{ }^{1}$ Yuanyuan $\mathrm{Wu},{ }^{3}$ Yinhua Su, ${ }^{2}$ Jianzhi Li, ${ }^{2}$ \\ Li Liao, ${ }^{2}$ and Daqi Liao $\mathbb{1}^{2}$ \\ ${ }^{1}$ Xiangya School of Public Health, Central South University, Changsha, Hunan 410001, China \\ ${ }^{2}$ School of Nursing, University of South China, Hengyang, Hunan 421001, China \\ ${ }^{3}$ Wuxi Mental Health Center, Wuxi 214000, China \\ Correspondence should be addressed to Huilan Xu; shisaisai433@sina.com
}

Received 2 December 2021; Accepted 27 December 2021; Published 24 February 2022

Academic Editor: Bhagyaveni M.A

Copyright (C) 2022 Rong Wang et al. This is an open access article distributed under the Creative Commons Attribution License, which permits unrestricted use, distribution, and reproduction in any medium, provided the original work is properly cited.

Rehabilitation intervention which refers to the functional training by caregivers with the aid of specialized nursing techniques and the progressive promotion of patients' training initiative, with the purpose of improving mobility and quality of life, is of great significance. The purpose of the study was to investigate the effect of the rehabilitation intervention-centered targeted nursing model on the cardiac function recovery and negative emotions in patients with acute myocardial infarction (AMI). A total of 120 AMI patients admitted to our hospital between January 2019 and January 2020 were selected as the study subjects and randomly divided into group A $(n=60)$ and group B $(n=60)$, in which the group B patients received routine nursing combined with rehabilitation intervention, while based on the treatment in group $\mathrm{B}$, the patients in group $\mathrm{A}$ underwent rehabilitation intervention-centered targeted nursing model. Then, the cardiac function indexes, negative emotion score, levels of risk factors for heart failure, complication rate (CR), and the quality of life (QOL) of the patients were compared between the two groups. The cardiac function indexes of the patients after nursing in group A were significantly better than those in group B $(P<0.001)$; the negative emotion scores of the patients after nursing in group A were significantly lower than those in group $\mathrm{B}(P<0.001)$; the levels of risk factors for heart failure of the patients after nursing in group A were significantly lower than those in group $\mathrm{B}$ $(P<0.001)$; the $\mathrm{CR}$ of the patients in group $\mathrm{A}$ at $15 \mathrm{~d}$ and $30 \mathrm{~d}$ after admission was significantly lower than that in group $\mathrm{B}$ $(P<0.05)$; the QOL scores of the patients after nursing in group A were significantly higher than those in group $\mathrm{B}(P<0.001)$. Rehabilitation intervention-centered targeted nursing model can optimize cardiac function, weaken the levels of risk factors for heart failure, reduce the incidence of complications, improve psychological conditions, and enhance the quality of life in AMI patients, which is worthy of application and promotion in clinical practice.

\section{Introduction}

The majority of AMI patients usually presents with myocardial ischemia and hypoxia, declined cardiac function, and impaired respiratory system, further leading to their weakened mobility; therefore, rehabilitation intervention which refers to the functional training by caregivers with the aid of specialized nursing techniques and the progressive promotion of patients' training initiative, with the purpose of improving mobility and quality of life, is of great significance [1-3]. However, current studies on the rehabilitation intervention for AMI patients in clinical practice have revealed that, due to the twin stressors of physical and psychological conditions, patients have low compliance with rehabilitation training; besides, the routine nursing model is less targeted and cannot relieve patients' negative emotions, so it has relatively limited effect on facilitating rehabilitation [4-7]. AMI patients' great suffering of severe pain at the onset of the disease combined with the oppressive hospital environment can result in their heavy psychological 
burdens, which may further aggravate or eventually come with an extremely obvious tendency of anxiety and depression, greatly hindering the progress of rehabilitation. At present, targeted nursing has become one of the focuses of clinical concern, and it considers patients' subjective complaints and actual conditions as the implementation basis of nursing measures, with the advantages of high pertinence and personalized services. Based on this, in order to investigate the effect of the rehabilitation intervention-centered targeted nursing model on AMI patients, a total of 120 AMI patients admitted to our hospital between January 2019 and January 2020 were selected as the study subjects, and the study results are summarized as follows.

\section{Materials and Methods}

2.1. General Information. A total of 120 AMI patients admitted to our hospital between January 2019 and January 2020 were selected as the study subjects and randomly divided into group A $(n=60)$ and group $\mathrm{B}(n=60)$. There were no significant differences in the general information of the patients between the two groups $(P>0.05)$, with research significance, as detailed in Table 1.

2.2. Inclusion Criteria. The inclusion criteria were as follows: (1) patients and their family members were informed of the purpose and process of the study and signed informed consent. (2) Patients met the AMI diagnostic criteria made by the American Heart Association [8, 9]. (3) Patients had the onset within twelve hours. (4) Patients' Killip classification of cardiac function was below IV. (5) This study was approved by the hospital.

2.3. Exclusion Criteria. The exclusion criteria were as follows: (1) patients had other organic diseases or complications such as pulmonary edema. (2) Patients had mental disorders or could not communicate with others. (3) $\mathrm{Pa}$ tients had lumbar fracture.

2.4. Methods. The group B patients received routine nursing combined with rehabilitation intervention, and the specific steps were as follows. (1) Nursing staff should pay much attention to the changes of patients' sign data to check whether there were signs of deterioration and provide patients with routine diet and medication guidance. (2) Nursing staff should ask the patients to lie in bed for a rest and provide physical nursing by the way of wiping patients' skin to perform the passive activity within one week of onset. After one week, the patients were encouraged to get up by themselves and perform stretching in semireclining positions. After two weeks, the patients were instructed to get out of bed to do some exercises, such as walking, and their walking time could be prolonged with the increase of rehabilitation intervention, and nursing staff should pay much attention to the occurrence of adverse reactions in patients.

Based on the treatment in group B, the patients in group A were given extra rehabilitation intervention-centered
TABle 1: Comparison of general information between the two groups.

\begin{tabular}{lcccc}
\hline Group & $\begin{array}{c}\text { Group A } \\
(n=60)\end{array}$ & $\begin{array}{c}\text { Group B } \\
(n=60)\end{array}$ & $X^{2} / t$ & $P$ \\
\hline Gender & 40 & 41 & 0.038 & 0.845 \\
Male & 20 & 19 & & \\
Female & & & & \\
\hline Age (years) & $32-74$ & $33-74$ & & \\
Age range & $51.21 \pm 6.20$ & $51.23 \pm 6.21$ & 0.018 & 0.986 \\
Average age & 32 & 33 & 0.034 & 0.855 \\
Hypertension & 12 & 13 & 0.051 & 0.822 \\
Cerebral & 25 & 24 & 0.035 & 0.853 \\
infarction & & & & \\
Diabetes mellitus & 25 & 24 & 0.035 & 0.853 \\
\hline Infarction location & 18 & 18 & 0.000 & 1.000 \\
Anterior wall & 10 & 8 & 0.261 & 0.609 \\
Inferior wall & 7 & 10 & 0.617 & 0.432 \\
Side wall & & & & \\
Others & 26 & 27 & 0.034 & 0.854 \\
Killip classification & 22 & 20 & 0.147 & 0.702 \\
I & 12 & 13 & 0.051 & 0.822 \\
II & & & &
\end{tabular}

targeted nursing model, and the specific steps were as follows. (1) Nursing staff should increase their own empathy to keep close to patients' mental status and timely carry out psychological counseling. Besides, in order to improve patients' cooperation with the rehabilitation, nursing staff should frequently tell patients the positive significance of rehabilitation training, adopt integrating or target systems to promote their motor initiative, and timely give patients encouragement and rewards after they reached their small goals. At the same time, when patients were undergoing rehabilitation training, nursing staff should explain the knowledge related to the disease timely and play some soothing music to relieve their anxiety and restlessness. (2) Before the initiation of nursing care, nursing staff should comprehensively grasp patients' clinical data, increase the frequency of communication, listen to their subjective appeal, and adjust nursing plans at any time according to patients' specific demands and actual conditions, so as to make the rehabilitation intervention program personalized. (3) Nursing staff should carry out rehabilitation within 24 hours of patients' onset by the way that patients changed their supine positions to semireclining positions which were then kept for half an hour, and then the patients returned to their original supine positions with the help of nursing staff. Such positionchanging exercises were required to be performed more than 3 times per day. After one day of patients' onset, nursing staff should tell patients the positive role of getting up from bed on their own to arouse their willingness to get up actively and help them perform physical stretching in semireclining positions. At two days after patients' onset, nursing staff should ask patients to carry out sitting training on bed and strengthen the exercise with enhanced rehabilitation intervention. At three days after patients' onset, the patients were asked to get out of the bed to stand for no 
less than 10 minutes per day, and additionally, they were encouraged to walk, and after four days, the patients should intensify the training in walking by prolonging walking distance [10-13]. (4) Nursing staff should encourage patients' family members to pay much attention to patients, effectively exerting the dual functions of professional nursing and family nursing.

\subsection{Observation Indexes}

(1) Levels of risk factors for heart failure: the levels of angiotensin II (Ang II), aldosterone (ALD), and B-type natriuretic peptide (BNP) were compared between the two groups

(2) CR: the rate of complications including arrhythmia and heart failure was compared between the two groups at $1 \mathrm{~d}\left(T_{1}\right), 15 \mathrm{~d}\left(T_{2}\right)$, and $30 \mathrm{~d}\left(T_{3}\right)$ after admission

(3) Cardiac function indexes: the left ventricular endsystolic dimension (LVESD), left ventricular enddiastolic dimension (LVEDD), left ventricular ejection fraction (LVEF), and early/late diastolic peak velocity of the left atrioventricular valve (E/A ratio) before and after nursing intervention were compared between the two groups

(4) Negative emotion score: the negative emotions of the patients were evaluated by the self-rating anxiety scale (SAS) and the self-rating depression scale (SDS), and higher scores indicated severer negative emotions in patients

(5) QOL: the QOL was evaluated by the QOL-C30 scale, whose items included emotional function, physical function, social function, role function, and cognitive function, and higher scores indicated better QOL

2.6. Statistical Treatment. The selected data processing software for this study was SPSS 20.0, and software selected to draw the pictures was GraphPad Prism 7 (GraphPad Software, San Diego, USA). Measurement data were tested by $t$-test, and enumeration data were tested by $X^{2}$ test. The differences had statistical significance when $P<0.05$.

\section{Results}

3.1. Comparison of Cardiac Function Indexes between the Two Groups. After nursing, the LVESD and LVEDD levels in group A were significantly lower than those in group B $(P<0.001)$, and the LVEF level and E/A ratio in group A were significantly higher than those in group $\mathrm{B}(P<0.001)$, as shown in Table 2.

3.2. Comparison of Negative Emotion Scores between the Two Groups. After nursing, the negative emotion scores in group A were significantly lower than those in group B $(P<0.001)$, as shown in Table 3.
3.3. Comparison of the Levels of Risk Factors for Heart Failure between the Two Groups. The levels of risk factors for heart failure after nursing in group A were significantly lower than those in group B $(P<0.001)$, as shown in Table 4.

3.4. Comparison of CR between the Two Groups. The CR in group $\mathrm{A}$ at $T_{2}$ and $T_{3}$ was significantly lower than that in group B $(P<0.05)$, as shown in Figures 1 and 2.

3.5. Comparison of the QOL after Nursing between the Two Groups. The QOL scores after nursing in group A were significantly higher than those in group $\mathrm{B}(P<0.001)$, as shown in Figure 3.

\section{Discussion}

AMI patients' great suffering of severe pain at the onset of the disease combined with the oppressive hospital environment can result in their heavy psychological burdens, which may further aggravate or eventually come with an extremely obvious tendency of anxiety and depression, greatly hindering the progress of rehabilitation [14-17]. Routine nursing care mainly focuses on the clinical efficacy of rehabilitation intervention, and nursing staff usually lay emphasis on the completion degree of functional training tasks and ignore timely psychological counseling, and thus, patients' depression cannot be addressed, and rehabilitation effect remains unsatisfactory. Better intervention effect can be achieved by enhancing the frequency of communication with patients, listening to their subjective appeals, and developing more targeted and personalized nursing measures for them [18-22]. The majority of AMI patients usually presents with myocardial ischemia and hypoxia, declined cardiac function, and impaired respiratory system, further leading to their weakened mobility; therefore, rehabilitation intervention which refers to the functional training by caregivers with the aid of specialized nursing techniques and the progressive promotion of patients' training initiative, with the purpose of improving mobility and quality of life, is of great significance.

In this study, the negative emotion scores of the patients in group A after nursing were significantly lower than those in group $\mathrm{B}(P<0.001)$ because nursing staff alleviates patients' anxiety and depression by introducing the knowledge related to the disease in detail and actively responding to the problems raised by the patients, so patients' adverse emotions are effectively relieved. Due to more positive emotions, an increased initiative to perform rehabilitation training, and the combination with rehabilitation intervention in the early stage of the disease in group A, the cardiac function indexes as well as the levels of risk factors for heart failure in group A after nursing were significantly better than those in group B $(P<0.001)$, and the $\mathrm{CR}$ at $15 \mathrm{~d}$ and $30 \mathrm{~d}$ after admission in group A was significantly lower than that in group B $(P<0.05)$, indicating that nursing can greatly improve patents' hypoxia tolerance and effectively promote the recovery of cardiac function and exercise capacity, with more desirable recovery effect. 
TABLE 2: Comparison of cardiac function indexes between the two groups $(\bar{x} \pm s)$.

\begin{tabular}{|c|c|c|c|c|c|c|}
\hline \multirow[t]{2}{*}{ Types } & \multicolumn{2}{|c|}{ Group A } & \multicolumn{2}{|c|}{ Group B } & \multirow{2}{*}{$\frac{t}{0.029}$} & \multirow{2}{*}{$\begin{array}{c}P \\
0.977\end{array}$} \\
\hline & Before nursing & $55.21 \pm 3.56$ & Before nursing & $55.23 \pm 4.01$ & & \\
\hline \multirow{3}{*}{ LVESD (mm) } & After nursing & $47.12 \pm 3.25$ & After nursing & $52.23 \pm 3.58$ & 8.186 & $\leq 0.001$ \\
\hline & $t$ & 13.000 & $t$ & 4.323 & & \\
\hline & $P$ & $\leq 0.001$ & $P$ & $\leq 0.001$ & & \\
\hline \multirow{4}{*}{ LVEDD (mm) } & Before nursing & $62.12 \pm 4.56$ & Before nursing & $62.23 \pm 4.65$ & 0.131 & 0.896 \\
\hline & After nursing & $50.23 \pm 3.98$ & After nursing & $58.11 \pm 3.54$ & 11.459 & $\leq 0.001$ \\
\hline & $t$ & 15.217 & $t$ & 5.461 & & \\
\hline & $P$ & $\leq 0.001$ & $P$ & $\leq 0.001$ & & \\
\hline \multirow{4}{*}{ LVEF (\%) } & Before nursing & $45.65 \pm 4.01$ & Before nursing & $45.78 \pm 4.02$ & 0.177 & 0.860 \\
\hline & After nursing & $59.12 \pm 5.78$ & After nursing & $52.89 \pm 5.79$ & 5.899 & $\leq 0.001$ \\
\hline & $t$ & 14.832 & $t$ & 7.813 & & \\
\hline & $P$ & $\leq 0.001$ & $P$ & $\leq 0.001$ & & \\
\hline \multirow{4}{*}{$\mathrm{E} / \mathrm{A}$ ratio } & Before nursing & $0.81 \pm 0.21$ & Before nursing & $0.82 \pm 0.20$ & 0.267 & 0.790 \\
\hline & After nursing & $2.01 \pm 0.32$ & After nursing & $1.35 \pm 0.36$ & 10.614 & $\leq 0.001$ \\
\hline & $t$ & 24.285 & $t$ & 9.969 & & \\
\hline & $P$ & $\leq 0.001$ & $P$ & $\leq 0.001$ & & \\
\hline
\end{tabular}

TABLE 3: Comparison of negative emotion scores between the two groups ( $\bar{x} \pm s$, points).

\begin{tabular}{lcccr}
\hline Group & \multicolumn{2}{c}{ SDS } & & SAS \\
& Before nursing & After nursing & Before nursing & After nursing \\
\hline Group A & $50.12 \pm 5.32$ & $30.26 \pm 2.12$ & $56.15 \pm 5.30$ & $35.11 \pm 2.87$ \\
Group B & $50.32 \pm 5.56$ & $38.45 \pm 3.68$ & $56.35 \pm 4.89$ & $43.12 \pm 4.85$ \\
$t$ & 0.201 & 14.938 & 0.215 & 11.010 \\
$P$ & 0.841 & $\leq 0.001$ & 0.831 & $\leq 0.001$ \\
\hline
\end{tabular}

TABLE 4: Comparison of the levels of risk factors for heart failure between the two groups $(\bar{x} \pm s, \mathrm{pg} / \mathrm{ml})$.

\begin{tabular}{|c|c|c|c|c|c|c|}
\hline \multirow[t]{2}{*}{ Types } & \multicolumn{2}{|c|}{ Group A } & \multicolumn{2}{|c|}{ Group B } & \multirow{2}{*}{$\begin{array}{c}t \\
0.404\end{array}$} & \multirow{2}{*}{$\begin{array}{c}P \\
0.687\end{array}$} \\
\hline & Before nursing & $160.21 \pm 13.56$ & Before nursing & $161.20 \pm 13.25$ & & \\
\hline \multirow{4}{*}{ Ang II } & After nursing & $110.20 \pm 10.55$ & After nursing & $130.35 \pm 10.58$ & 10.446 & $\leq 0.001$ \\
\hline & $t$ & 22.547 & $t$ & 14.093 & & \\
\hline & $P$ & $\leq 0.001$ & $P$ & $\leq 0.001$ & & \\
\hline & Before nursing & $172.41 \pm 15.45$ & Before nursing & $172.42 \pm 15.56$ & 0.004 & 0.997 \\
\hline \multirow{3}{*}{ ALD } & After nursing & $132.58 \pm 10.50$ & After nursing & $156.32 \pm 12.59$ & 11.217 & $\leq 0.001$ \\
\hline & $t$ & 16.516 & $t$ & 6.231 & & \\
\hline & $P$ & $\leq 0.001$ & $P$ & $\leq 0.001$ & & \\
\hline \multirow{4}{*}{ BNP } & Before nursing & $565.21 \pm 74.21$ & Before nursing & $566.50 \pm 75.41$ & 0.094 & 0.925 \\
\hline & After nursing & $160.25 \pm 30.58$ & After nursing & $240.65 \pm 42.59$ & 11.878 & $\leq 0.001$ \\
\hline & $t$ & 39.081 & $t$ & 29.144 & & \\
\hline & $P$ & $\leq 0.001$ & $P$ & $\leq 0.001$ & & \\
\hline
\end{tabular}

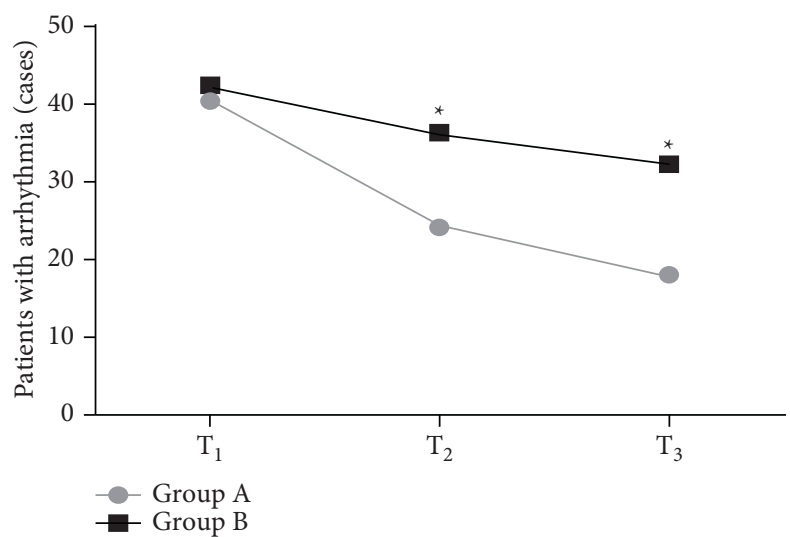

FIGURE 1: Comparison of the incidence of arrhythmia between the two groups. Note: the abscissa represented $1 \mathrm{~d}\left(T_{1}\right), 15 \mathrm{~d}\left(T_{2}\right)$, and $30 \mathrm{~d}$ $\left(T_{3}\right)$ after admission. The incidence of arrhythmias at $T_{1}$ was $66.7 \%(40 / 60)$ in group A and 70.0\% (42/60) in group B. The incidence of arrhythmias at $T_{2}$ was $40.0 \%(24 / 60)$ in group A and 60.0\% (36/60) in group B. The incidence of arrhythmias at $T_{3}$ was $30.0 \%(18 / 60)$ in group $\mathrm{A}$ and $53.3 \%(32 / 60)$ in group $\mathrm{B} .{ }^{*} P<0.05$. 


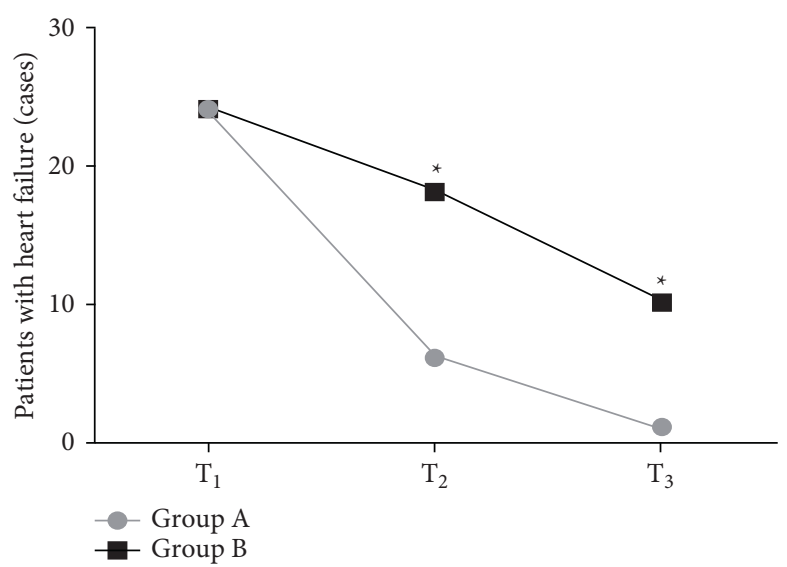

Figure 2: Comparison of the incidence of heart failure between the two groups. Note: the abscissa represented $1 \mathrm{~d}\left(T_{1}\right), 15 \mathrm{~d}\left(T_{2}\right)$, and $30 \mathrm{~d}$ $\left(T_{3}\right)$ after admission. The incidence of heart failure at $T_{1}$ was $40.0 \%(24 / 60)$ in group A and $40.0 \%(24 / 60)$ in group B. The incidence of heart failure at $T_{2}$ was $10.0 \%(6 / 60)$ in group A and 30.0\% (18/60) in group B. The incidence of heart failure at $T_{3}$ was $1.7 \%(1 / 60)$ in group A and $16.7 \%(10 / 60)$ in group B. ${ }^{*} P<0.05$.

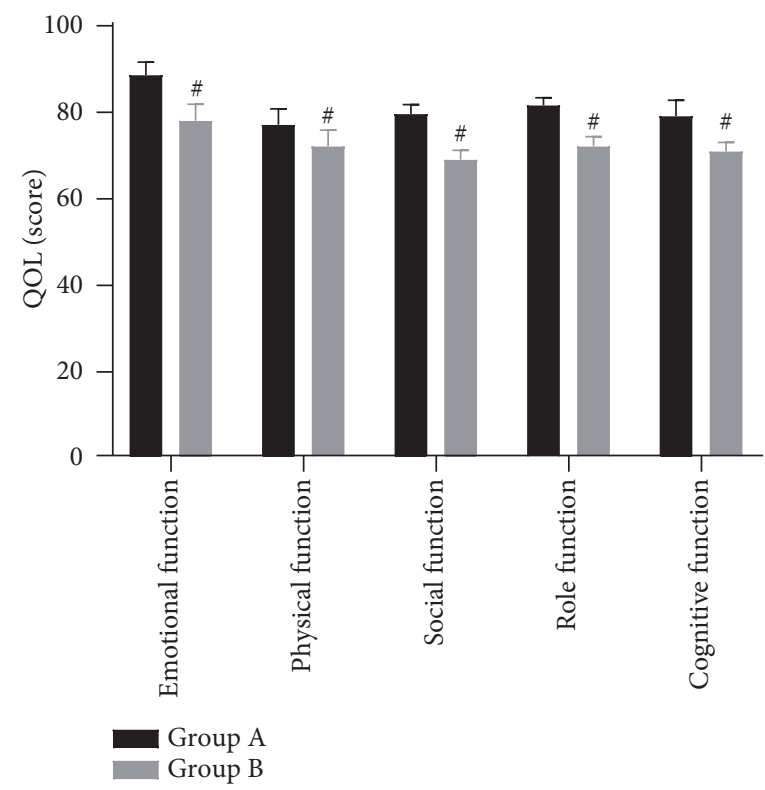

FIgURE 3: Comparison of the QOL after nursing between the two groups ( $\bar{x} \pm s$, points). Note: the abscissa represented emotional function, physical function, social function, role function, and cognitive function. The emotional function scores were $86.21 \pm 4.22$ points in group A and $75.02 \pm 5.41$ points in group B. The physical function scores were $74.20 \pm 5.21$ points in group A and $69.10 \pm 5.32$ points in group B. The social function scores were $77.81 \pm 3.02$ points in group A and $67.23 \pm 3.00$ points in group B. The role function scores were $80.12 \pm 2.41$ points in group A and $70.23 \pm 3.16$ points in group B. The cognitive function scores were $76.20 \pm 5.20$ points in group A and $69.10 \pm 3.00$ points in group B. ${ }^{\#} \mathrm{P}<0.001$.

\section{Conclusion}

Moreover, as a result of increased frequency of communication with patient's family members and better mastery of rehabilitation knowledge, the QOL scores of the patients in group A after nursing were significantly higher than those in group $\mathrm{B}(P<0.001)$, and the functional scores in all aspects of the patients in group A were better than those in group $B$, confirming the positive effect of the targeted nursing intervention. Scholar Tracy Sue in her study pointed out that the scores of emotional function, physical function, social function, role function, and cognitive function in the research group, in which the patients receive the functional rehabilitation-centered targeted nursing model, are $86.61 \pm 3.22$ points, $75.20 \pm 5.02$ points, $77.89 \pm 4.21$ points, $82.31 \pm 3.41$ points, and $76.11 \pm 4.90$ points, respectively, which are all significantly higher than those in the reference group where the patients underwent routine rehabilitation intervention $(P<0.001)$, indicating that patients in the research group have a better quality of life and recovery [23]. Her study results are in line with the findings concluded in our study.

In conclusion, rehabilitation intervention-centered targeted nursing model can effectively enhance cardiac function and relieve negative emotions of AMI patients, which is worthy of application and promotion in clinical practice. In 
the future, we need more cases to ensure the reliability of the experimental results. Our experiment still has many limitations.

\section{Data Availability}

The datasets used and/or analyzed during the current study are available from the corresponding author upon reasonable request.

\section{Disclosure}

Rong Wang is the single first author.

\section{Conflicts of Interest}

The authors declare that they have no conflicts of interest.

\section{References}

[1] R. S. Passinho, C. C. Primo, M. Fioresi, M. M. L. da Nóbrega, M. A. G. Brandão, and W. G. Romero, "Elaboration and validation of an ICNP ${ }^{\circledR}$ terminology subset for patients with acute myocardial infarction[J]," Revista da Escola de Enfermagem da USP, vol. 53, Article ID e03442, 2019.

[2] A. R. Zullo, R. Ofori-Asenso, M. Wood et al., "Effects of statins for secondary prevention on functioning and other outcomes among nursing home residents[J]," Journal of the American Medical Directors Association, vol. 21, no. 4, pp. 500-507, 2020.

[3] D. Hetclova, M. Hutyra, J. Precek et al., “4034Distance from hospital as an early marker of outcomes in out of hospital cardiac arrest acute myocardial infarction survivors treated with endovascular mild therapeutic hypothermia[J]," European Heart Journal, vol. 40, no. Supplement_1, 2019.

[4] I. Popescu, N. Sood, S. Joshi, P. Huckfeldt, J. Escarce, and T. K. Nuckols, "Trends in the use of skilled nursing facility and home health care under the hospital readmissions reduction program: an interrupted time-series analysis[J]," Medical Care, vol. 57, no. 10, pp. 757-765, 2019.

[5] D. E. Twigg, Y. Kutzer, E. Jacob, and K. Seaman, "A quantitative systematic review of the association between nurse skill mix and nursing-sensitive patient outcomes in the acute care setting," Journal of Advanced Nursing, vol. 75, no. 12, pp. 3404-3423, 2019.

[6] H. Babamohamadi, H. Kadkhodaei-Elyaderani, A. Ebrahimian, and R. Ghorbani, "The effect of spiritual care based on the sound heart model on the spiritual health of patients with acute myocardial infarction[J]," Journal of Religion and Health, vol. 59, no. 3, pp. 2638-2653, 2020.

[7] S. Hoschar, L. Albarqouni, and K. H. Ladwig, "A systematic review of educational interventions aiming to reduce prehospital delay in patients with acute coronary syndrome[J]," Open Heart, vol. 7, no. 1, Article ID e001175, 2020.

[8] J. G. Jollis, C. B. Granger, T. D. Henry et al., "Systems of care for ST-segment-elevation myocardial infarction: a report from the American heart association's mission: lifeline," Circulation: Cardiovascular Quality and Outcomes, vol. 5, no. 4, pp. 423-428, 2012.

[9] R. A. Chazal and S. R. Houser, "American college of cardiology and American heart association," Circulation, vol. 134, no. 24, pp. 2028-2029, 2016.
[10] J. A. Rolnick, J. M. Liao, E. J. Emanuel et al., "Spending and quality after three years of Medicare's bundled payments for medical conditions: quasi-experimental difference-in-differences study[J]," BMJ, vol. 369, Article ID m1780, 2020.

[11] E. Maria, K. H. Ngerud, B. Margareta, S. S. Lawesson, A. Strömberg, and I. Thylén, "Interaction between telenurses and callers with an evolving myocardial infarction: consequences for level of directed care[J]," European Journal of Cardiovascular Nursing, vol. 18, no. 7, pp. 545-553, 2019.

[12] W. S. Oliveira, J. R. Soares Júnior Júnior, S. R. O. Maier et al., "Perspective of nursing professionals on the discharge of patients suffering from recurrent myocardial infarction," Scientific Electronic Archives, vol. 13, no. 11, p. 69, 2020.

[13] Y. Kashiwagi, "A case of acute myocardial infarction during chemotherapy of advanced rectal cancer," Nurse Media Journal of Nursing, vol. 8, no. 2, p. 96, 2019.

[14] W. Widiyaningsih and E. Kusyati, "Hemodinamik pamidrpk," Journal of Holistic Nursing Science, vol. 6, no. 1, pp. 22-27, 2019.

[15] S. Noureddine, N. Y. Dumit, and H. Maatouk, "Patients' knowledge and attitudes about myocardial infarction[J]," Nursing and Health Sciences, vol. 22, no. 3, 2019.

[16] S. Gupta, F. D. Zengul, G. K. Davlyatov, and R. WeechMaldonado, "Reduction in hospitals' readmission rates: role of hospital-based skilled nursing facilities[J]," Inquiry: A Journal of Medical Care Organization, Provision and Financing, vol. 56, Article ID 004695801881799, 2019.

[17] M. M. Pashaki, Y. Dousti, and B. Mirzaian, "Modeling of cardiac function of patients with acute myocardial infarction based on Type-D personality: mediating role of negative emotions[J]," Journal Of Nursing And Midwifery Sciences, vol. 6, no. 2, p. 63, 2019.

[18] A. San-Juan-Rodriguez, Y. Zhang, M. He, and I. Hernandez, "Association of antidementia therapies with time to skilled nursing facility admission and cardiovascular events among elderly adults with alzheimer disease[J]," JAMA Network Open, vol. 2, no. 3, Article ID e190213, 2019.

[19] C. Nymark, P. Henriksson, A.-C. Mattiasson, F. Saboonchi, and A. Kiessling, "Inability to act was associated with an extended delay prior to care-seeking, in patients with an acute myocardial infarction," European Journal of Cardiovascular Nursing, vol. 18, no. 6, pp. 512-520, 2019.

[20] A. Shajrawi, A. M. Al-Smadi, G. K. Al-Shawabkeh, H. S. Aljribeea, and H. Khalil, "Impacts of treatment modalities on physical activity after first acute myocardial infarction in Jordan," Dimensions of Critical Care Nursing, vol. 38, no. 6, pp. 284-292, 2019.

[21] A. Protopapas and E. Lambrinou, "Cultural factors and the circadian rhythm of ST elevation myocardial infarction in patients in a Mediterranean island[J]," European Journal of Cardiovascular Nursing, vol. 18, no. 7, pp. 562-568, 2019.

[22] A. Elbadawi, H. M. A. Ahmed, I. Y. Elgendy et al., "Outcomes of acute myocardial infarction in patients with rheumatoid arthritis[J]," The American Journal of Medicine, vol. 133, no. 10 , pp. $1168-1179,2020$.

[23] S. Tracy, "Can more targeted nursing accelerate the recovery of patients with lower acute myocardium? [j]," Journal of the American Medical Directors Association, vol. 20, no. 1, 20219. 